\title{
PERSPECTIVA CRÍTICA DE LA INVESTIGACIÓN EN LA ESCUELA \\ GRADUADA BEATRIZ LASSALLE: EL CASO DE LA GÉNESIS Y DESARROLLO DEL TRABAJO SOCIAL EN PUERTO RICO
}

\section{Jesús M. Cabrera Cirilo ${ }^{1}$}

Resta todavia mucho por hacer, nuestra esperanza queda puesta en los trabajadores sociales del futuro que llevarán la profesión hasta el pleno desarrollo de su madurez.

Carmen Rivera de Alvarado (1939)

\section{Resumen}

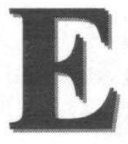

1 Trabajo Social en Puerto Rico, tiene el desafío de fortalecer el debate sobre sus fundamentos filosóficos, teóricos e históricos. Se puede reconocer que el debate contemporáneo en Trabajo Social viene manteniendo una constante discusión académico-profesional sobre los fundamentos que componen la práctica y la investigación. Siendo así, en este espacio, haremos un vistazo por algunos de los trabajos finales de graduación que representan un valioso caudal de hallazgos del recorrido histórico de nuestro quehacer. Para este análisis utilizaremos la perspectiva crítica, que de acuerdo a Marx no significa rechazo al conocimiento anterior, sino la superación de este por medio del análisis a un nivel más profundo.

\footnotetext{
${ }^{1}$ Trabajador Social y estudiante de maestría en Trabajo Social de la Universidad de Costa Rica.
} 
Descriptores: investigación, trabajo social, historia, metodología, política social, imperialismolcolonialismo.

\section{Abstract}

Social Work in Puerto Rico has the challenge of strengthening the debate between its philosophical, theoretical and historical basis. It can be acknowledge in nowadays debate of Social Work that there is a constant academic -professional discussion about the basis between praxis and investigation. In this article we make an all around examination of works that represent an invariable volume of findings that offer an historic course to our occupation. In the analysis we used the critical perspective, which for Marx is not a rejection of previous knowledge but an improvement by means of a more profound knowledge.

Key words: research, social work, history, methodology, social policy, imperialism \ colonialism.

\section{Introducción}

El Trabajo Social en Puerto Rico, tiene el desafío de propiciar el debate sobre sus fundamentos filosóficos y teóricos partiendo del compromiso con el fortalecimiento teórico-crítico de la profesión y a nuestra responsabilidad histórico-social de re-definir las acciones, visiones, perspectivas, marcos teóricos, ontológicos y epistemológicos. Aunado, el momento histórico que le ha tocado vivir a las nuevas generaciones de profesionales en Trabajo Social, presenta nuevos retos que necesitan una amplia discusión de los aspectos ético-políticos, teórico-metodológicos y técnico-operativos de nuestro quehacer.

Se puede reconocer que el debate latinoamericano en Trabajo Social viene manteniendo una constante discusión académico-profesional sobre los cimientos que componen la formación profesional. Importante por demás es, reconocer los productos de las discusiones y debates que desde comienzos del Movimiento de 
Reconceptualización ${ }^{2}$ se han interiorizado en el gremio. Estas discusiones trascendieron las fronteras de los países del hemisferio. $\mathrm{La}$ influencia de los cuestionamientos, reflexiones y reclamos afectaron y repercutieron, de diferentes maneras, en muchas de las Escuelas de Trabajo Social latinoamericanas. Fueron muchos los esfuerzos por atender las cuestiones referentes a la profesión y desde varias Escuelas surgieron diversas propuestas, intentando atender los requerimientos que la propia realidad les engendró e interpuso. A las actuales generaciones de profesionales del Trabajo Social ha llegado la depuración de todo este acontecimiento histórico. Algunos temas son continuados, y a su vez, ampliados por la contribución detallada de las peculiaridades del desenvolvimiento histórico-social de cada país. ${ }^{3}$

En la actualidad, se están llevando a cabo varias investigaciones sumamente importantes particularmente para profesionales de trabajo social de América Latina. La discusión histórico-crítica del origen, naturaleza e instrumentalidad de ésta profesión en los diferentes países, circunscribiendo el análisis al desarrollo de las relaciones sociales en el sistema de producción capitalista. ${ }^{4}$ La ruptura que se logra con esta perspectiva fundamenta un análisis crítico-teórico que

\footnotetext{
${ }^{2}$ Se entiende como Movimiento de Reconceptualización, el proceso surgido desde la década de 1960 en Latinoamérica, en el cual se plantea la superación de la segmentación metodológica en el quehacer profesional del Trabajo Social. En Puerto Rico comenzó en la década del 70 y no fue impulsada por las escuelas de Trabajo Social, sino por un grupo profesional, Asociación Nacional de Trabajadores Sociales (ANTS). Sobre este tema ver; Alayón (2005).

${ }^{3}$ El hilo conductor es el saqueo, la expropiación, el militarismo, el colonialismo y la explotación de los recursos naturales, en definitiva del capitalismo dependiente a los diferentes imperios. En Puerto Rico, al igual que otros países de la región centroamericana, el desarrollo del capital ha estado ligado a las intervenciones directas de la burguesía y milicia norteamericana, la historia así lo demuestra.

${ }^{4}$ En oposición con la tesis anterior, ésta no formula una simple contextualizaciòn de la profesión. Aquí, no se pretende una 'historia del Trabajo Social', sino más bien, el entendido de la profesión en un contexto histórico específico y bajo condiciones particulares. En esta perspectiva el Trabajo Social es aprehendido como un producto de la división socio- técnica del trabajo, que cumple un papel dentro del orden socioeconómico (Iamamoto, 1997). En el sistema capitalista la profesión mantiene la funcionalidad de sostener y reproducir las relaciones sociales que se extrapolan de la lucha de clases dentro del sistema económico.
} 
proporciona una dirección analítica que supera las lecturas superficiales y unidireccionales que hegemónicamente han caracterizado la profesión. Lo realizado por trabajadores y trabajadoras sociales, que se adhieren a la perspectiva crítica e histórica fundamentada en el marxismo, es el intento de continuación de la corriente más progresista y de avanzada de la Reconceptualización, pero superando sus limitaciones. Por lo tanto, en este artículo se pretende continuar con lo comenzado por docentes, profesionales y estudiantes que han producido exploraciones relacionadas con el rescate histórico del origen y desarrollo de nuestra profesión, en este caso Puerto Rico.

Nos parece importante hacer varias aclaraciones sobre este trabajo. En el mismo se presenta es una síntesis de las reflexiones realizadas para la justificación del trabajo final del autor: Capitalismo imperialista estadounidense y génesis del Trabajo Social en Puerto Rico (1930 al 1960). En un principio, lo fundamental era identificar aquellos trabajos, publicados o no, que pudieran ser utilizados para problematizar las tesis explicativas del origen del Trabajo Social en Puerto Rico. Además, los textos que pudieran ofrecer información documental de las políticas sociales, proyectos político-económicos, espacios de trabajo y la labor que ejercieron las profesionales durante la consolidación de la hegemonía imperialista en el archipiélago puertorriqueño. Así que para la construcción de este trabajo, la revisión bibliográfica hecha se enfocó en todos aquellos trabajos finales de graduación realizados durante los últimos 63 años en la Escuela Graduada Beatriz Lasalle, Universidad de Puerto Rico. ${ }^{6}$ Sin embargo, la impresión generada por los trabajos realizados en la Escuela Graduada Beatriz Lassalle durante varias décadas, sobre todo

\footnotetext{
${ }^{5}$ Propuesta de investigación defendida ante el postrado en Trabajo Social de la Universidad de Costa Rica, para optar por el grado de Magíster Scientiae en Trabajo Social con énfasis en investigación.

${ }^{6}$ Aunque en Puerto Rico hay varias Escuelas Graduadas de Trabajo Social, estas son de reciente fundación. Por las determinaciones ontológicas y fundamentales del objeto de estudio, para poder hacer una trayectoria histórica de la concepción de la génesis del Trabajo Social puertorriqueño hay que recurrir a los debates profesionales originarios y estos se encuentran contenidos en la producción intelectual de las profesionales de principio de Siglo XX, desarrollados en la Escuela Graduada de Trabajo Social Beatriz Lassalle.
} 
las del período del 40-50, llevó a analizar con más profundidad algunos aspectos importantes de la investigación en Trabajo Social, como por ejemplo, la cuestión metodológica. Siendo así, en este espacio sólo se expondrán las disertaciones de maestría ${ }^{7}$ que de algún modo han tratado la problemática de la génesis, origen o naturaleza del Trabajo Social en Puerto Rico. Se excluyen las publicaciones recientes, revistas profesionales y las producciones latinoamericanas que han desarrollado el tema en otros países.

\section{Análisis de disertaciones relacionadas con la génesis y desarrollo del Trabajo Social en Puerto Rico}

Las disertaciones analizadas fueron aqueellas que se entiende contribuyen a la aprehensión de la trayectoria histórica del Trabajo Social en Puerto Rico. Hasta el momento todos los textos revisados son conducentes al grado de maestro y maestría en Trabajo Social. ${ }^{8}$ Se utilizaron varios parámetros para el proceso discriminatorio. Primero se precedió al examen de la organización de la lista de tesis de la Biblioteca de Trabajo Social Monserrate Santana de Palés. Luego se escogieron las que discutieran la historia de la profesión a base de un espacio laboral determinado, por ejemplo, la historia de la profesión en instituciones gubernamentales, política social, entre otras. Como indicación final, la exposición sigue la lógica organizativa y temática utilizada por la biblioteca, en el primer listado los temas se encuentran ordenados alfabéticamente y las tesis en forma cronológica, por año de presentación. En el segundo y tercer listado están por año de presentación.

\footnotetext{
${ }^{7}$ En Puerto Rico no existe el grado de licenciatura, los grados académicos son bachillerato, maestría y doctorado.

${ }^{8}$ En las tesis de grado realizadas durante los primeros años de la Escuela Graduada se recurre a la categoría de maestro y no de maestría, porque la Escuela Graduada Beatriz Lassalle estuvo adscrita a la Facultad de Educación.
} 
Tesis realizadas en los años 1945-1979 ${ }^{9}$

I. Empleo, desempleo y asuntos del trabajo

La primera tesis revisada fue la de Hilda V. Maldonado Díaz, Bernardo Muñiz Arocho, José R. Ramírez Polo, Mario Roche Velásquez, Julia E. Rodríguez Bernguer, Ludia M. Rosa Pérez, Néstor Rosado Malpica, Sara M. Saliva Guillot, Adminda Sierra Martínez, Francisco Torres Rivera, Eulogio Vélez Velásquez, Zulma Vélez González (1969). ${ }^{10}$ Servicio Social en la Puerto Rican Cement Company Incorporada división de San Juan, por reacción al Servicio Social de enero de 1967 a enero de 1969. Esta investigación es de carácter exploratorio-descriptivo a posteriori. Tuvo como finalidad determinar las funciones del trabajador social en la empresa estatal Puerto Rican Cement Company, para lo que se preparó y suministró una planilla al personal de la empresa. En esta tesis no se trabaja la historia del Trabajo Social en Puerto Rico. No obstante, en su justificación los autores y las autoras desarrollan una pequeña introducción a la situación económica imperante en el momento del estudio. Además, hacen referencia al proceso de cambio políticoeconómico implantado en la Isla, el proyecto de Manos a la Obra, modelo de desarrollo que llevó al territorio puertorriqueño de la producción agrícola a la rápida industrialización.

Un dato sobresaliente es que los y las tesistas, en su preocupación por atender con mayor rigurosidad el tema, y con la intención de obtener más información sobre el área de Trabajo Social industrial, escribieron a diferentes escuelas de Servicio Social, específicamente a México, América Central, Suramérica y España. Según lo detallado en la investigación, recibieron contestación de la Escuela de Trabajo Social de la Universidad Autónoma de Hidalgo, México, Escuela de Trabajo Social de Nuevo León, Monterrey, México, Universidad

\footnotetext{
${ }^{9}$ Los períodos que se establecen son los utilizados en los listados de organización de la biblioteca de Trabajo Social Beatriz Lassalle, esto significa, por ejemplo que el primer listado de tesis comienza en el 1945 hasta el 1979, el próximo desde 1979 hasta el 1989.

${ }^{10}$ Como se puede notar, se colocan todos los nombres completos, se hace esto para reconocer la labor realizada por todos y todas las colegas que durante todas estas décadas trabajaron para fortalecer la profesión.
} 
Pontificia Bolivariana, Facultad de Trabajo Social, Medellín, Colombia y la Escuela de Servicio Social del Salvador.

Los investigadores y las investigadoras verificaron, y concluyeron que las funciones desempeñadas por la trabajadora social en esta industria, respondieron a determinadas necesidades psico-sociales del personal de la industria. Entonces, la práctica en dicha empresa de producción estatal, se dirigía a la adaptación y resignación de la fuerza de trabajo a los requerimientos de la producción, y posiblemente a la mediación entre los conflictos obrero-patronales.

\section{Problemas comunales, participación y vida comunal}

En el trabajo realizado por Aída G. Becerra Nazario (1963), Funciones del trabajador social en un programa de comunidades planificadas. El objeto de estudio fue, la evaluación de las funciones de nueve trabajadores sociales que sirvieron a las comunidades urbanas y rurales planificadas de la Administración de Programas Sociales del Estado Libre Asociado de Puerto Rico, durante el año fiscal 1958-59. Mantuvo el propósito de conocer las funciones que desempeñaban los trabajadores sociales que servían a la Administración de Programas Sociales durante la delimitación temporal, con un diseño de carácter exploratorio. La autora desarrolló dos planillas para ser suministrada a las trabajadoras sociales seleccionadas, un total de nueve.

En la justificación, Becerra (1963) contextualiza el tema estableciendo la invasión norteamericana como punto de partida para una nueva distribución y división territorial de la Isla. Sostiene que, la Administración de Programas Sociales del Departamento de Agricultura se instaura con la ley número 216 del 15 de mayo de 1948. Entre las acciones que este organismo desarrolló, se encuentran la reinstalación de agregados y la construcción de viviendas en comunidades rurales dispuestas por el titulo V de la Ley de Tierras; la distribución y la administración de fincas individuales de acuerdo con el titulo VI, la administración del programa de granjas de hogares seguros y el programa de educación cooperativa. Pero menciona que durante los primeros tres años de funcionamiento, 1948-1950, la 
administración de programas sociales tuvo a su cargo el desarrollo de los programas anotados. Inició, además, en forma experimental los programas de hogares a bajo costo, de pequeñas industrias, de abastecimiento de agua a las comunidades mediante el hincamiento de pozos, de construcción de letrinas y de mejoramiento físico de las comunidades (Becerra, 1963).

Este dato es indicativo de la funcionalidad de dicha política social, indudablemente la meta programática era crear mejores condiciones de vida, para la fuerza trabajadora excedente ${ }^{11}$ aglutinada en las áreas metropolitanas durante la expansión industrial. Es sin lugar a dudas, una política desarrollista relacionada esencialmente con el proceso de industrialización-urbanización de las áreas metropolitanas. Por otro lado, ilustra la actuación que profesionales de trabajo social que debían ayudar a las familias servidas en su adaptación y su ajuste de las nuevas condiciones de vivienda y trabajo; orientar a los agregados en cuanto al conocimiento de la Ley, a sus responsabilidades hacia la agencia y de la agencia hacia ellos. También hacían investigaciones de agregados, sorteos de parcelas, organización de comités de viviendas y orientación a familias con problemas (Becerra, 1963).

La anterior referencia, deja claro la labor realizada por profesionales de trabajo social en la implantación de la mencionada política social. Básicamente, el quehacer cotidiano profesional tenía como dirección adaptar la población a las nuevas instituciones formadas, y a sus exigencias, esencialmente fungiendo como mediadores entre el Estado y la Sociedad Civil. En conclusión, esta investigación ofrece información valiosa para identificar los objetivos de la política social, la población foco de la intervención, y por supuesto, la ocupación de los y las profesionales en su operacionalización.

\footnotetext{
${ }^{11}$ Fuerza de trabajo excedente es una categorización marxista para identificar aquella población que no tiene trabajo, pero que está lista para ser incorporada a la fuerza laboral. En la teoría social marxista es importante esta población, ya que es la mano de obra que abarata los costos de la que está empleada.
} 
III. Profesión de Trabajo Social, evaluación del adiestramiento profesional y trabajadores sociales

El primer trabajo analizado bajo esta categoría fue realizado por; Maria Virginia Honda (1955). Estudio del Colegio de Trabajadores Sociales de Puerto Rico 1940-1954. Según la autora, su pesquisa es un estudio de actitudes, se refiere a la parte subjetiva de la conducta humana, en otras palabras una indagación de opiniones verbalizadas. ${ }^{12}$ Para lograr esto, la investigadora creó una planilla dirigida a rescatar de las trabajadoras sociales de Puerto Rico una auto-evaluación de la relación de colegiados y colegiadas con el Colegio de Trabajadores Sociales durante los años 1940-1954.

Esta tesis, resuelve algunos problemas de ubicación histórica de la génesis del Colegio de Trabajadores Sociales de Puerto Rico. Es además, sumamente ilustrativa porque hay en ella un recuento historiográfico de asuntos relacionados a la organización profesional, especialmente los conflictos relacionados a la formación profesional según el lugar de estudio; el contraste entre profesionales que se educaron en la Isla versus los que alcanzaron grados académicos en los Estados Unidos. Es trascendente porque se desarrolló a 14 años de la fundación del Colegio, lo que aporta una aproximación inmediata de los asuntos determinantes en la consolidación del gremio, expuestos con un gran acervo documental-bibliográfico. Igualmente, Honda (1955) ofrece lineamientos analíticos de los proyectos que durante este período se implantaron, ya que expone, en un pequeño recuento, los reclamos del colectivo al gobierno estadounidense para la programación e implantación de política social en el territorio.

Bajo este mismo índice temático, se encuentra la investigación realizada por; Krimilda López Aviles y Doris R. Lugo de Toro (1960), Conocimientos y actitudes de un número de médicos sobre las

\footnotetext{
${ }^{12}$ En la actualidad podríamos ubicarla bajo la categoría de cualitativa, sin embargo, denota una combinación mixta de datos cualitativos y cuantitativos. Esto es significativo, ya que pone en tela de juicio la actual bifurcación; cualitativa o cuantitativa. En este caso, recordemos que estamos ante una tesis del 1955, no hay tal división y lo que sugiere es que en la misma se define la investigación con la técnica específica utilizada para el logro de la misma.
} 
funciones de los trabajadores sociales en agencias gubernamentales de salud en Puerto Rico. Según las autoras, el diseño de investigación es uno clásico experimental ex-post-facto. El propósito del estudio fue determinar, con el uso de métodos objetivos, los conocimientos y las actitudes de un número de médicos sobre las funciones de los trabajadores sociales en agencias de salud. Las autoras consideraron como una limitación, la falta de una publicación sobre la historia del Trabajo Social. Según ellas, por esta limitación, se les hizo difícil el recrear fidedignamente los inicios de la profesión en la Isla.

El documento adscribe el Trabajo Social con los servicios médicos a partir de los años 1923-1924, cuando el Negociado de Bienestar Social requiere sus servicios para la atención de las personas participantes de la agencia gubernamental. Dicha agencia tenía bajo su dirección clínicas pre-natales e infantiles, enfermeras visitantes y de servicios sociales, los cuales consistían en la provisión de recursos económicos a familias necesitadas. Para administrar los servicios sociales, ofrecidos bajo esta dependencia, contratan en 1924, a una trabajadora social estadounidense, Nellie Foster. Este dato, confirma que a la altura de la década del 20 aún no comienza la reproducción nativa de trabajadores y trabajadoras sociales, sino que se importan un número considerable de profesionales estadounidenses durante el proceso de consolidación de las instituciones en la colonia. ${ }^{13}$ Además, esta investigación identifica situaciones históricas determinantes para la reconstrucción de la trayectoria profesional. Por ejemplo, las políticas sociales que comienzan a ser ejecutadas desde la esfera colonial, y la formación de estructuras político-gubernamentales,

\footnotetext{
${ }^{13}$ Es con la llegada de la Cruz Roja Americana que comienzan a ofrecerse espacios para la labor voluntaria con las familias de los militares de la Primera Guerra Mundial. Es la Cruz Roja quien tomó la iniciativa en proveer talleres académicos para su personal, los cuales se inician en el verano del 1923 en la Universidad de Puerto Rico, bajo la iniciativa de Beatriz Lassalle (Ruíz, 1997:50). Ya para el año 1928 es reconocida la profesión en la isla en el Departamento de Instrucción Pública en las Segundas Unidades Rurales. Esta es una propuesta en donde no sólo se les enseña a los niños las materias ordinarias, sino que además son focos centrales de educación comunitaria. Pero es en la década del 1930, cuando comienza realmente la especialización y reproducción del Trabajo Social, período cuando inician los cursos profesionales en la Universidad de Puerto Rico.
} 
cimientos de los que más adelante serán las que cobijarán a gran parte de profesionales de Trabajo Social.

Por otro lado, Alba Miranda Villamil, Olga Morena de Rodríguez, Gloria Josefina Otero, Ana Isabel Tous Gómez y Ligia Vélez Hernández (1964), investigaron El papel del trabajador social en la acción social. El diseño de la investigación es exploratorio, a posteriori de una sola celda y en un sólo tiempo. El propósito del estudio fue, determinar si las trabajadoras sociales que prestaron servicios profesionales en al División de Bienestar Público del Departamento de Salud de Puerto Rico, participaron en el procesos de acción social. En la justificación, las autoras realizan un análisis sumamente interesante sobre la política social, y la acción social de profesionales del trabajo social. Aseguran que las personas entrevistadas no conciben la manera en que deben participar en la fomentación, creación, y revisión de programas, no obstante, sí la necesidad de que el Trabajo Social participe en la formulación de la política social.

En el texto hay una continua referencia a las pioneras de la profesión en los Estados Unidos, nombran a Jane Adams, y Mary Richmond. Le adjudican a éstas, el inicio de la acción social como método para mejorar las condiciones de vida de las poblaciones desvalidas. Hacen un reconocimiento tácito de que tanto las pioneras en Estados Unidos como en la Isla, desempeñaron un papel de gran significado al ganar la aceptación de la comunidad y al elevar los niveles de vida de ambos pueblos (Miranda et. al, 1963: 20). Alba y Olga definen política social como la acción:

...dirigida a satisfacer la necesidad del hombre por disfrutar de una seguridad económica, de una vivienda decente y de conservar la estabilidad de sus relaciones familiares por medio de la provisión de servicios y de una persistente indagación, tanto de las causas del fracaso, como de los métodos para evitarlos (Miranda et. al, 1963: 32).

Según esta conceptuación, la política social es una herramienta de producción que ayuda a superar los obstáculos interpuestos por la 
cuestión económica. Contradictoriamente, reducen el problema económico a la iniciativa que cada individuo tenga, si no se superan los problemas económicos es porque el colectivo familiar no ha podido reestructurar sus metas. En esta tesis, además se percibe a profesionales de trabajo social como un grupo profesional preparado para comprender el significado de la acción social. Es la primera ocasión, que algún trabajo de tesis hace referencia a la formulación de la política social como posible espacio de trabajo.

Otra indagación es la realizada por Rita Córdova, María del Mar García Rodríguez, Blanca Hernández, Miriam Matos, Carmen Mejía Valle, Ada Olabarrieta, Julio Ortega Candelaria, Antonia Rivera, Eduardo Viruet Correa y Yolanda Zayas (1970); Participación de los trabajadores sociales en asuntos controvertibles relativos al bienestar social. 1969-1970. El estudio es exploratorio descriptivo y tuvo la intención de determinar si los trabajadores y las trabajadoras sociales de Puerto Rico participan en los asuntos controvertibles del país. Para alcanzar el objetivo, el equipo de investigación creó un instrumento ${ }^{*}$ de observación estructurado. En la justificación sostienen:

A tono con la filosofía de la profesión consideran al trabajador social como un activista cuya función debe ser vital para la promoción de cambios fundamentales en la sociedad. Estiman que estos profesionales no deben eludir la confrontación con los problemas sociales. Así pues, la misión del trabajador social es descubrir, plantear y envolverse en todos aquellos asuntos controvertibles que muchas veces se ocultan o soslayan dentro de la sociedad misma y que pueden resultar en perjuicio de la clientela (Córdova, et. al. 1970:1).

En esta investigación la motivación es el activismo político, y la profesión es entendida en su relación con el cambio social. No se trabaja históricamente el asunto, ni se hace referencia al contexto histórico, aunque mencionan lo conflictivo del momento histórico. Este trabajo final de graduación se diferencia de los anteriormente, por su carácter político. Es sin lugar a dudas, un intento por llevar a otro nivel la investigación en Trabajo Social y la crítica social. Sobre 
todo, la función y toma de decisiones de las profesionales en sus labores cotidianas.

En esta sección de la profesión de trabajo social se puede ubicar también el estudio realizado por Carmen Pilar Almodóvar Tirado, Efraín Ayala Medina, Advilda Bajandas Zayas, Wendell Boneta López, Georgina Bueno Delgado, Azalya Corujo Rodríguez, Ana L. Cucurella, Rafael Figueroa Villafañe, Alice Doris González Hernández, Arcadio González Brown, Enrique González Polanco, Rosa Marta Jacob Habitt, Helga Vanessa Nazario Torres, Migdalia Pérez Plaza (1971), que lleva como titulo Estudio sobre el código de ética del trabajador social. Es uno de carácter exploratoriodescriptivo. Tuvo como propósito, determinar el conocimiento que tenían los trabajadores y las trabajadoras sociales de Puerto Rico residentes en el área metropolitana de San Juan, acerca del Código de Ética. En esta tesis, el equipo de investigación desarrolla un análisis introductorio sobre la cuestión de la ética. Para estos, un código de ética "tiene valor en tanto y en cuanto se aplica a diario por los profesionales en todos los campos en que estos desempeñan sus funciones" (Almodóvar, et. al, 1971: 4).

Aquí se establece la ética, como aquella que es práctica, y no política. Aunque, continuamente se hace referencia a la justicia social, como meta de intervención ética, la misma es subordinada a la inserción del 'inadaptado' a la sociedad. En cuanto a la explicación de lo que es conceptualmente el Trabajo Social, utilizan a la trabajadora social norteamericana Helen Northen, que indica:

El valor último que guía a la práctica del trabajo social es que cada individuo deberá tener las oportunidades para realizar sus potencialidades para vivir en la forma que sean personalmente satisfactorias y socialmente útiles y deseables. La más profunda derrota personal sufrida por seres humanos consiste en la diferencia entre lo que fue capaz de llegar a ser y lo que no ha llegado a ser en realidad [...] el propósito del trabajo social relacionado directamente con los valores primarios es el mejoramiento psicosocial de los individuos y de las instituciones y condiciones ambientales. El concepto 
del funcionamiento psicosocial se refiere a la interacción de las personas en sus situaciones sociales y ambientales. El trabajo social tiene interés en saber las actitudes de las personas hacia sí mismo y hacia otras, sus sentidos adecuados e identidad personal y el acoplamiento de sus valores como guías hacia la realización cabal de sus potencialidades (Almodóvar, et. al, 1971:7).

Este trabajo refleja, en primer lugar, la ética desde valores morales, en segundo, la lógica funcionalista social. Desde esta perspectiva teórica, el Trabajo Social es entendido como un vehículo que debe ayudar a re-insertar las personas desviadas y marginadas a la sociedad, asistiéndolos en la socialización estructural. Bajo esta matriz aparecen las estructuras y las clases sociales como producto de las propias determinaciones naturales, superiores y por encima de los individuos. En síntesis, los autores visualizan la naturaleza de la profesión desde su fundamento conservador.

\section{Temas varios (misceláneos) ${ }^{14}$}

El primer trabajo revisado en esta categoría es el de, Concepción Bertrán de Bassó (1949); Proyecciones sociales del Titulo V de la Ley de Tierras de Puerto Rico. La investigación es una exploratoriadescriptiva. Tuvo como propósito; analizar los efectos de las disposiciones del Titulo V de la Ley numero 26 del 12 de abril de 1941, en las familias parceleras de Buenaventura, Carolina, Puerto Rico, con el fin de determinar si se lograron los objetivos de dicha ley.

La exploración tiene como objetivo el establecer por medio de la investigación, si la Ley de Tierras, logró su cometido en cuanto a la repartición de fincas de "agregados". Para alcanzar los objetivos

${ }^{14}$ Como se anotó al principio de este componente del trabajo, las categorías de organización de las investigaciones son las de la lista original usado en la Escuela Graduada de Trabajo Social Beatriz Lassalle. Ahora bien, este título (misceláneo) incluye varios temas entre los que se destaca el de política pública, hay que recordar los parámetros utilizados para la discriminación temática y específica de la investigación. 
trazados, la investigadora preparó una planilla para recoger la opinión de las familias que residían en la comunidad. Concepción (1949) define a una persona agregada como jefe de familia que resida en la zona rural, cuyo hogar se encuentre en casa y terrenos ajenos o en casa propia levantada en terreno ajeno.

La definición anterior es importante porque es un análisis de la política social distributiva implantada durante el período del 1940 al 1950, básicamente consistió en la repartición de tierras a personas que no eran propietarias del suelo donde vivían. Aunque la investigadora no realiza un análisis detallado de las repercusiones de la ley, es sin lugar a dudas un acercamiento continuo a la evaluación de lo programado por los gobiernos coloniales de la Isla. En la introducción Bertrán de Bassó (1949) recoge, de la exposición de motivos de la Ley, lo que en esencia es la política social redistributiva:

[....] es parte integrante del propósito moral, de dignidad y libertad económicas encarnado en la política pública de la Legislatura, el facilitar los medios para que desaparezca de Puerto Rico la clase de 'agregados' o sea de trabajadores agrícolas esclavizados por el hecho de no ser dueños ni siquiera del pedazo de terreno donde tienen sus hogares; y a tal efecto la Legislatura declara el derecho fundamental humano de todos los seres que viven exclusivamente de trabajar la tierra, de ser por lo menos dueños de un pedazo de esa tierra que les sirva para levantar sobre él, con la entera inviolabilidad que garantizan las leyes al hogar del ciudadano, su propio hogar, libertándolo así de coacciones y dejándolo libre para vender su trabajo en negociación justa y equitativa (pág. 1). 
Efectivamente, esta política atiende una situación particular de la "cuestión social colonial"15; la inmigración interna de la población rural que se asienta en la zona industrial-urbana en busca de mejores condiciones de subsistencia. En resumen, se rescata de la indagación la descripción de la situación en que vivían estas poblaciones, y de la política social dirigida a ellas.

Otro tema tratado en esta sección es realizado por: Marmen Mercedes Barrios de Cruz, Lourdes del R. Carrión Pagán, María del Carmen Hernández Ricoff, Iraida Lugo Lebrón, Ana Isabel Meléndez Ibáñez, María Yolanda Pérez Almodovar, Sheila Rodríguez Rodríguez y Haydeé Suárez Rivera (1978); Aportación y utilización por los diferentes organismos y agencias gubernamentales -Administración de Corrección División de Bienestar Público, Departamento de Servicios Contra la Adicción, Departamento de Instrucción Pública, Departamento del Trabajo, Administración de Tribunales, Departamento de Salud, Departamento de la Vivienda, Colegio de Trabajadores Sociales de Puerto Rico y Escuela Graduada de Trabajo Social, - de los esfuerzos de investigación hechos en la Escuela Graduada de Trabajo Social Beatriz Lassalle de la Universidad de Puerto Rico entre los años 1945-1976.

Según las autoras, este es un estudio diagnóstico-descriptivo, fundamentado en el análisis de contenido. Para lograr el objetivo de la investigación crearon un formulario guía, que se completaba a medida que se revisaban las tesis. El propósito de la indagación fue, conocer

\footnotetext{
15 "La cuestión social" es la consecuencia del surgimiento del proletariado como clase antagónica, que simultáneamente, va adquiriendo fuerza en sus reclamos, tanto reivindicativos como organizativos. En su avance organizativo, trabajadores y trabajadoras se reconocen como clase, que tiene intereses dispares con relación a los capitalistas. Pero de igual manera, la clase socialmente dominante reconoce, y advierte, el carácter revolucionario de la organización de la clase trabajadora. Entonces, "la cuestión social" es una terminología para designar un modo específico de pauperización; el de la sociedad burguesa. Es una conceptualización que define la confrontación que se desenvuelve de la contradicción capital \trabajo, en fin, de la lucha de clases. Con la utilización del concepto "cuestión social colonial" se pretende abarcar (o por lo menos intentar) la particularidad de la relación política-económica de Puerto Rico con los EE.UU., en resumen captar la lucha de clases en el territorio colonial.
} 
si los resultados de las investigaciones generadas en la Escuela Graduada de Trabajo Social, fueron incorporados o tomados en consideración en las agencias públicas.

Este amplio documento es fundamental para la carrera de Trabajo Social, sobre todo para la investigación. Lo esencial de este trabajo académico es la preocupación de las tesistas de conocer si verdaderamente las instituciones gubernamentales y educativas estaban incorporando los hallazgos y recomendaciones generadas desde la Escuela Graduada. Es indiscutible la aportación que esta tesis tiene para la profesión, claro producto del intento de ruptura de la Reconceptualización. Lo comprueba la utilización de varios autores identificados con el Movimiento reconceptualizador, como AnderEgg. Además, las descripciones socio-contextuales son basadas en vasta documentación, lo que le imprime un aditamento adicional. Por todo lo anterior, debe ser lectura obligatoria para todo estudiante de Trabajo Social. Más aún, cuando hay una recurrente defensa de la investigación en la profesión para continuar con la profundización del entendido de la problemática social en la Isla.

\section{Trabajo médico-social}

En esta categoría se encuentra el trabajo producido por: Aida Mirayes Sastre, Hilda Pérez Guardiola, José Robles González y Antonio S. Rodríguez (1959), Funciones de los trabajadores médico sociales del Distrito Sanitario de Río Piedras. El objetivo principal del trabajo final de graduación fue, comprobar científicamente las demandas del servicio médico social en el Distrito Sanitario de Río Piedras e identificar las tareas de los profesionales en sus espacios laborales.

Para completar el estudio los y las tesistas, revisaron los informes estadísticos mensuales, a lo que le llaman estudio de tiempo. También crearon dos cuestionarios, uno para los trabajadores sociales seleccionados y otro para ser completado por el director médico del centro. En la contextualización histórica, que antecede la investigación, sostienen que la intención de establecer el servicio médico social en la Isla se remonta a los años del 1923-1924. Según los datos propuestos en este espacio, fue bajo la tutela del 
Departamento de Sanidad que se creó el Negociado de Bienestar Social bajo la Ley número 38. Declaran que fue para estos años que el gobierno de Puerto Rico, empezó a preocupase por la situación de salud de la población. Según los autores, es ya en el 1926 que se establece el primer centro de salud público en Río Piedras cuando el gobierno insular solicita los servicios médico sociales, por la situación del alto índice de mortalidad infantil.

En este período, los primeros desempeños estuvieron dirigidos a evaluar las condiciones socio-económicas para ayudar a solucionar sus problemas inmediatos. Sin embargo, y como relatan los investigadores, surgieron para intervenir con la situación maternoinfantil. A medida que se fueron ampliando los servicios y cambiando las estrategias gubernamentales en la atención de diferentes situaciones, las intervenciones de profesionales fueron también ampliando su esfera de ejecución.

Como puede observarse, esta producción es anterior a la hecha por Krimilda Lopez, y Doris R. Lugo, inicialmente revisada. Aunque tienen muchas concordancias, se diferencian esencialmente en los objetos de estudios. En el primer caso, querían conocer la opinión de los médicos sobre la labor de trabajadores y trabajadoras sociales en los espacios de salud. En este caso, el interés era identificar las tareas de los profesionales en espacio laboral específico, Río Piedras.

\section{Trabajo social escolar}

La disertación más notoria de esta sección se encuentra la hecha por Carmen Lydia Noriega de Santa y Candelaria Crespo de Rosa (1956), El trabajo social escolar en Puerto Rico durante los años de 19501955. El objetivo primordial fue examinar las funciones del trabajador social escolar o maestro visitante, en servicio activo durante los años del 1950 al 1953. Aunque no se establece qué tipo de investigación es, por la revisión del trabajo se entiende que es de carácter exploratorio-descriptivo. Es en resumen, un estudio de opinión, para estos fines se utilizó un cuestionario para examinar el Trabajo Social en el sistema educativo por medio de la opinión de los y las profesionales. 
En la introducción contextualizan el tema históricamente. En el capítulo II incluyen un recuento histórico de la educación en los Estados Unidos, en el que se explica como diferentes profesiones van incorporándose al sistema educativo. Utilizan diferentes autores norteamericanos para aseverar que el Trabajo Social Escolar en los Estados Unidos comienza en el año 1906 y 1907, concretamente en las ciudades de Boston, Hartford y en la ciudad de Nueva York. Para explicar las labores que ejercieron los trabajadores y las trabajadoras sociales en las escuelas rurales en el país, las autoras acuden a colegas que participaron de los programas escolares para conocer sobre los comienzos del mismo. En el texto, identifican a las escuelas de las comunidades como los centros de trabajo de los profesionales, asegurando que desde este espacio desarrollaron los proyectos rurales $\mathrm{y}$ urbanos.

\section{Vivienda}

En esta categoría se destaca la tesis desarrollada por; Lucila Alejandro Ortiz, Lourdes Rivera Correa y Dolores Sandín de López (1960); Problemas de ajuste en 60 familias trasladadas de áreas de nivel socio-económico bajo a la urbanización pública. Es un estudio exploratorio con la finalidad de entender sus condiciones de vida antes y después del traslado al residencial público. Se fundamentó con la recolección de las opiniones de residentes. Incorporan un recuento historiográfico de la política urbanística desarrollada para la región metropolitana, pero desasociada del contexto económico-político en el cual surge la operacionalización de la política. Se presenta como un proceso espontáneo, o peor aún como una propuesta lógica de desarrollo.

Disertaciones realizadas durante el periodo de 1979 al $1989^{16}$

Carmen Cancel Reyes, José R. Carrión Medina, Mercedes Marqués Llompart, Angel L. Meaux Pereda, Wanda L. Rivera Márquez, Carol

\footnotetext{
${ }^{16}$ En las subsiguientes listas, cambia la lógica organizativa de las tesis, ya no son organizadas por temas, sino por año de presentación.
} 
Rodríguez Cintrón (1987), Análisis de la política desempleo en Puerto Rico: el caso de la administración de derecho al trabajo, llevaron a cabo una investigación histórica. Utilizaron análisis de contenido y entrevistas a personas involucradas en el proceso de la creación de la Administración del Trabajo. Se propusieron conocer las políticas públicas de desempleo durante 1940-1968. Se reconoce como la primera pesquisa que proporciona un marco económicopolítico general para estudiar una acción particular, la política pública desarrollada contra el desempleo. A partir de los hallazgos, se pueden establecer históricamente cuáles fueron las políticas públicas dirigidas a intervenir con el problema del desempleo en la Isla. En el documento sintetizan los datos más significativos de las diferentes leyes contra el desempleo. Es un esfuerzo de exploración histórica, pero no hacen un análisis crítico de lo obtenido.

Otra investigación histórica es la de Mayra Enid Díaz Díaz, Leslia Lisette Parzons Pagán y Maribel Ramos Vera (1987); Análisis histórico de la supervisión y la administración en la profesión de trabajo social en Puerto Rico entre los años de 1920 hasta el 1979. La metodología utilizada fue la historia oral y revisión documental. El trabajo tuvo el objetivo de determinar históricamente la fecha cuando comenzó la supervisión del Trabajo Social en Puerto Rico. En este trabajo final de graduación, las autoras ofrecen datos significativos para el estudio de la génesis profesional. Según lo certificado, ya para el año 1930 se encontraban varias trabajadoras sociales administrando los programas sociales, demostrando que, efectivamente, en Puerto Rico más allá de implantar la política social a través del servicio directo, también gerenciaron y administraron proyectos del gobierno imperialista en la colonia.

\section{Disertaciones realizadas durante el periodo de 1990-2008}

La única disertación que se consideró para ser incluida en esta sección fue la producida por Lydimar Garriga Vidal, Damaris Negrón Rodríguez (2008) La formación neoliberal en el Trabajo Social. Este trabajo de investigación tuvo la aspiración de analizar la filosofía, misión, metas, objetivos y contenido de los cursos medulares de la Escuela Graduada Trabajo Social Beatriz Lasalle (EGTSBL) a la luz 
de las políticas educativas neoliberales. El análisis se centró en dos categorías de análisis, la educación como mercancía y la fragmentación del conocimiento en la formación profesional de Trabajo Social. Esta investigación fue exploratoria con un diseño mixto y con un enfoque cualitativo. La técnicas utilizadas en la disertación fueron: análisis de documentos y un cuestionario con preguntas abiertas y semiabiertas.

Esta indagación es, sin lugar a dudas, la primera tesis que aborda su análisis desde la crítica marxista, incluyendo el debate históricocrítico. En suma, es actualmente un verdadero intento de superar las limitaciones de la educación neoliberal. Específicamente el trabajo realizado por estas profesionales, es importante porque contribuye al debate interno de la profesión en Puerto Rico, problematizando aspectos de la educación graduada; la educación neoliberal. En él se problematizan, además, las formas explicativas de la génesis del Trabajo Social en Puerto Rico, concluyendo que la manera de explicar la profesión ha sido endógenamente. ${ }^{17}$

Las trabajadoras sociales, incorporaron el debate contemporáneo histórico-crítico para sostener sus posiciones en cuanto la historia de la profesión en la Isla. En suma, recurren a la contrastación de lo que la Escuela Graduada propone como fundamento filosófico de enseñanza (visión crítica del humanismo) y lo que realmente logran con la programación de los cursos que se ofrecen a los egresados. Para las investigadoras las dos categorías que definen la educación en el contexto neoliberal son: la visualización de la educación como una mercancía y la fragmentación del conocimiento. El trabajo tiene muchas fortalezas y es en esta última categoría de análisis una de las más sobresalientes, ya que pone en discusión la división del conocimiento característico de la influencia del positivismo en las ciencias sociales.

${ }^{17}$ Esta tesis propone que la génesis del Trabajo Social se explica por sí misma. Es decir, la profesión es expuesta desde "la historia del Trabajo Social", y no como un proceso histórico. Bajo esta tesis se hace una abstracción de la profesión, desvinculándola de la totalidad social. Además, la profesión se relaciona con las otras formas de ayudas filantrópicas y se visualiza como una continuación de estas protoformas. 


\section{Análisis general}

Este trabajo revela varias cuestiones transcendentales para el estudio de la génesis y desarrollo profesional en Puerto Rico. Primeramente, se advierte una restricción que sobresale de todas las demás. La falta de un trabajo que ubique y describa historiográficamente los primeros años de la formalización de profesionales de trabajo social. La bibliografía relacionada con las labores realizadas durante los primeros años de la proliferación de la carrera se encuentra fragmentada en pedazos autónomos. Es decir, que todos los datos son específicos en cuanto un área particular de intervención.

De hecho, es por medio de las primeras investigaciones de grado que se puede establecer, parcialmente, cuales fueron los espacios determinados de trabajo. La labor realizada, en las primeras investigaciones de la Escuela Graduada de Trabajo Social siempre tuvieron como prioridad e intencionalidad entender su quehacer, en proyectos sociales precisos o en las agencias gubernamentales, lo que demuestra dos cosas. En primer lugar, la necesidad de evaluar continuamente sus intervenciones y en segundo, la gran vinculación de la Escuela con los gobiernos de turno.

En las tesis el quehacer cotidiano de las trabajadoras sociales es identificado con las acciones paliativas del gobierno colonial. Aclarando lo anterior, se describen las actuaciones de profesionales y su relación con las estructuras gubernamentales de salud, bienestar social, la niñez, creadas por las diferentes administraciones estadounidenses, pero no son criticadas, así tampoco el trabajo concreto realizado en ellas. Esto a pesar de que no solo estuvimos en la formulación, diseño e implantación de gran parte de las políticas socio-económicas, sinó que además en la gerencia de los proyectos político-económicos durante la consolidación de las estructuras coloniales en formación. En este caso la profesión y su desarrollo estuvieron ligados indudablemente a los vaivenes históricos y a las necesidades particulares de la metrópoli.

Las investigaciones de grado evidencian un gran apego a la descripción de los fenómenos sociales y no hacia una explicación que 
considere las determinaciones fundacionales de la 'cuestión social colonial'. La realidad es fragmentada en problemas sociales focalizando, parcializando y abstrayendo su comprensión como totalidad histórica. Es decir, no se considera que lo entendido como problemas sociales sea la expresión viva de la reproducción de las relaciones sociales en el capitalismo, en nuestro caso colonial. Además, en la mayoría de las tesis no se hace un análisis de las políticas sociales implantadas en la Isla por el gobierno de E.U. como parte del proceso de colonización y adecuación al modo de producción capitalista monopólico. Siendo la excepción la tesis sobre la formación neoliberal en Trabajo Social.

Por otra parte, la confrontación de la lucha de clases en el territorio luego de la invasión estadounidense, la lucha nacionalista por la instauración de un gobierno independiente, la pauperización de las grandes mayorías, y la respuesta del imperialismo \colonialismo a los reclamos realizados, ${ }^{18}$ no se evidencian en las indagaciones como condiciones socio-históricas que proveyeron los espacios de intervención. No obstante, se identifica la inclusión analítica de la situación económico-política como necesaria para entender aspectos de la profesión. La génesis del Trabajo Social se muestra como surgimiento de la espontaneidad, o como parte evolutiva de la caridad o filantropía del gobierno y la burguesía local, haciendo una relación lineal entre la caridad y la profesión. Es como si la realidad social, y la historia en la cual se fragua, no mantuvieran contradicciones inmanentes al devenir de los procesos económicos, políticos y culturales.

\footnotetext{
${ }^{18}$ En otras palabras, las políticas socio-económicas son desarrolladas para intervenir con la acumulación histórica de las contradicciones creadas por la implantación del modo de producción capitalista en el país invadido; la pauperización, la militarización, la explotación de la clase trabajadora por la monopolización de la producción, la expropiación de la tierra, y la imposición de su jurisprudencia. Ante la 'cuestión social colonial' el gobierno imperialista \ colonialista responde, con un doble frente de ataque; el primer frente es el de guantes de seda con 'ayudas' canalizadas por una naciente clase profesional, el segundo es el garrote utilizado contra aquellos que no estaban de acuerdo con el régimen. Desde ese entonces este es el "modus operandi" de los represores imperiales junto con sus lacayos de la seudo- burguesía 'nacional'.
} 
En relación al fundamento teórico-metodológico, en las disertaciones revisadas se entrelazan el método y la metodología. ${ }^{19}$ El método se visualiza como la forma de intervenir con el objeto de estudio, estableciendo previamente las categorías de análisis y el instrumento de recolección de los datos. Los investigadores y las investigadoras metódicamente recurren a la construcción de preguntas, para de algún modo, validar las investigaciones, lo que demuestra el empirismo característico del cientificismo metodológico, derivación de la influencia ejercida por el positivismo; lo verdadero es aquello que puede ser demostrado utilizando el método científico, el cual es neutral y objetivo. ${ }^{20}$ Aunque se reconoce que las tesis tienen limitaciones en este aspecto, por estar enmarcadas en el positivismo, hay que destacar el esfuerzo, la meticulosidad y la convicción con que se realizaron las investigaciones de grado, sobre todo de las realizadas durante los años 40 al 50. De ellas se desprende el compromiso con el fortalecimiento profesional y el gran manejo de las técnicas que fueron utilizadas.

Otro dato importante de reseñar es la alta incidencia de tesis exploratorias-descriptivas. Para nuestra profesión, una de carácter eminentemente interventiva, pareciera que la descripción de lo inmediato es suficiente para lograr una real aproximación al problema u objeto de estudio, hecho que se demuestra (al menos en Puerto

\footnotetext{
${ }^{19}$ Netto aclara esto; [...] se confunde método con pauta de intervención, o sea, como estrategia de intervención, de acción. Es evidente que las estrategias de acción son intercambiables, ellas son seleccionables, pero eso no es método; son un conjunto de procedimientos practico-empíricos, practico-inmediatos, implementados para alcanzar determinados resultados. Evidentemente existe un conjunto de estrategias a elegir, y el profesional es tanto más competente cuanto más controle ese conjunto sobre el cual puede optar. Sin embargo eso no es metodología (Netto, 2000:75).

${ }^{20} \mathrm{El}$ positivismo ha sido una de las corrientes más influyentes de finales del siglo XIX y principios del XX. Se entiende como un sistema filosófico basado en la experiencia y el conocimiento empírico de los fenómenos naturales, en el cual la Metafísica y la Teología son sistemas de conocimientos imperfectos e inadecuados. El desarrollo de la ciencia en los últimos siglos le ha permitido posicionarse como una disciplina del 'verdadero conocimiento', ya que lo aceptado y creíble, es primordialmente lo que se puede comprobar por algún proceso positivo. Desde esta perspectiva, lo social es considerado como ecualización de la naturaleza, dicha herencia fundamentada en el pensamiento Comtiano, asume que la interpretación básica del movimiento social está sujeta a leyes físicas invariables.
} 
Rico) con la continua realización de este tipo de indagación. El inconveniente es que éstas sólo atienden la superficialidad, la representación fenomenológica de la contradicción capital-trabajo, no su origen. ${ }^{21}$ La situación se complica, ya que la descripción de lo inmediato, lo que se nos devela cotidianamente, contiene las mediaciones generales de la contradicción capital-trabajo pero no las particulares, aquellas que nos ofrecen los vínculos dialécticos de su superación-transformación. Consideramos que este tipo de lectura es problemática para una profesión como Trabajo Social porque provoca que sus intervenciones sean remediativas-paliativas, no transformadoras.

Recapitulando, para realizar una problematización del entendido de la génesis del Trabajo Social en Puerto Rico, debemos contrastar las posiciones de las diferentes generaciones que han contribuido al desarrollo de la profesión en la colonia norteamericana. Lo que en este artículo se presentó, es una muestra de las posturas asumidas por las colegas que han aprehendido el reto de proyectar la carrera de Trabajo Social en la Isla. Es indiscutible que una profesión que tiene a su haber más de 80 años de ejercicio social, contiene en su trayectoria divergencias, inflexiones, estancamientos, evoluciones, trascendencia, avances y retrocesos, lo que hace de su análisis historiográfico una hazaña rica y $\sin$ igual.

Para finalizar, la falta de un abordaje histórico-crítico cimentado en la teoría y filosofía de la dialéctica materialista marxista, que desarrolle un examen crítico de la génesis y desarrollo del Trabajo Social en Puerto Rico utilizando las contradicciones propias del proceso de colonización-imperialista como condiciones sociales, políticas, económicas y culturales que se desarrollan durante la expansión capitalista, ha provocado vacíos importantes en nuestra profesión. Por ello se cuestiona, ¿Cómo proyectar la profesión hacia el futuro si no

${ }^{21}$ La contradicción capital-trabajo es el fundamento del sistema capitalista. Es la lucha de clases generada por la expropiación de los medios de producción, por un lado los trabajadores que subsisten de vender la única mercancía que tienen para intercambiar con el capitalista; la fuerza de trabajo, y por otro la burguesía. Siendo así, entre el trabajo y el capital hay una contradicción inherente, el capitalista explota a los trabajadores y las trabajadoras para obtener mayor ganancia, plusvalía. 
conocemos las condiciones socio-históricas que incidieron en la génesis profesional? ¿Cómo podemos cuestionar nuestros espacios de legitimación si no partimos del análisis de las contradicciones que nos constituyen? ¿Qué podemos esperar de la profesión, si no se cuestiona el modo de producción capitalista, representado en la dominación colonialista en Puerto Rico? Además, ¿Qué papel ha jugado el Trabajo Social durante ese proceso? Nos parece, que las respuestas a estos cuestionamientos nos ayudaran a superar muchas de nuestras deficiencias profesionales.

Entendemos que para poder superar nuestros vacíos, los cuales muchos y muchas reconocemos, debemos asumir la autocrítica como fundamento neurálgico de la profesión. Esto nos pone una disyuntiva polémica, o nos envolvemos en resolver nuestras limitaciones teóricas debatiendo internamente o seguimos postergando, lo que ya es inevitable, el fortalecimiento de la educación profesional hegemónicamente crítica. Si optamos por lo último, de seguro comprometeremos el futuro de la carrera subordinándola a la continua disminución de espacios, tanto laborales como sociales. Por lo anterior, es que la actualización del tema desde un trabajo académicocrítico e incluyendo el debate contemporáneo en Trabajo Social es en la actualidad primordial, necesario y oportuno. 


\section{Referencias}

Alayón, Norberto. (2005) (Coord.). Trabajo Social latinoamericano, A 40 años de la Reconceptualización. Buenos Aires, Argentina: Espacio Editorial.

Alejandro, Lucila, Rivera, Lourdes \& Sandín, Dolores. (1960). Problemas de ajuste en 60 familias trasladadas de áreas de nivel socio-económico bajo a la urbanización pública. Tesis de maestría no publicada, Escuela Graduada de Trabajo Social Beatriz Lassalle, Universidad de Puerto Rico, en Río Piedras.

Almodóvar Tirado, Carmen Pilar; Ayala Medina Efraín; Bajandas Zayas, Advilda; Boneta López, Wendell; Bueno Delgado, Georgina; Corujo Rodríguez, Azalya; Cucurella, Ana L.; Figueroa Villafañe, Rafael; González Hernández, Alice Doris; González Brown, Arcadio; González Polanco, Enrique; Jacob Habitt, Rosa Marta; Nazario Torres, Helga Vanesa y Pérez Plaza, Migdalia. (1971). Estudio sobre el Código de Ética del trabajador social. Tesis de maestría no publicada, Escuela Graduada de Trabajo Social Beatriz Lassalle, Universidad de Puerto Rico, en Río Piedras.

Barrios de Cruz, Marmen Mercedes; Carrión Pagán, Lourdes del R.; Hernández Ricoff, María del Carmen; Lugo Lebrón, Iraida; Meléndez Ibáñez, Ana Isabel; Pérez Almodovar, María Yolanda; Rodríguez Rodríguez; y Suárez Rivera, Haydeé. (1973). Aportación y utilización de los diferentes organismos y agencias gubernamentales - Administración de Corrección, División de Bienestar Público, Departamento de Servicios Contra la Adicción, Departamento de Instrucción Pública, Departamento de Salud, Departamento de la Vivienda, Universidad de Puerto Rico, Colegio de Trabajadores Sociales - de los esfuerzos de investigación hechos en la Escuela Graduada de Trabajo Social Beatriz Lassalle entre los años 1945 y 197. Tesis de maestría no publicada, Escuela Graduada de Trabajo Social Beatriz Lassalle, Universidad de Puerto Rico, en Río Piedras. 
Becerra Nazario, Aida. (1963). Funciones del trabajadores social en un programa de comunidades planificadas. Tesis de maestría no publicada, Escuela Graduada de Trabajo Social Beatriz Lassalle, Universidad de Puerto Rico, en Río Piedras.

Bertrán de Bassó, Concepción. (1949). Proyecciones sociales del Titulo $V$ de la Ley de Tierras de Puerto Rico. Tesis de maestría no publicada, Escuela Graduada de Trabajo Social Beatriz Lassalle, Universidad de Puerto Rico, en Río Piedras.

Borgiani, Elisabete \& Montaño, Carlos. (Coord.). (2000). La política social hoy. Sao Paulo, Brasil: Cortez Editora.

Borgiani, Elisabete \& Montaño, Carlos. (2000). Metodología y Servicio Social. Sao Paulo, Brasil: Cortez Editora.

Borgiani, Elisabete; Guerra, Yolanda \& Montaño, Carlos. (Coord.). (2003): Servicio Social crítico. Sao Paulo, Brasil: Cortez Editora.

Burgos Ortiz, Nilsa M. (2001). Pioneras de la profesión de Trabajo Social en Puerto Rico. San Juan: Publicaciones Puertorriqueñạs.

Castro, Manrique. (1982). De apóstoles a agentes de cambio, el Trabajo Social en la historia latinoamericana. Lima, Perú: Ediciones CELATS.

Córdova Campos, Rita; García Rodríguez, María del Mar; Hernández, Blanca; Matos, Miriam; Mejía Valle, Carmen; Olabarrieta,Ada; Ortega Candelaria, Julio; Rivera, Antonia; Viruet Correa, Eduardo y Zayas, Yolanda. (1970). Participación de los trabajadores sociales en asuntos controvertibles relativos al bienestar social. Tesis de maestría no publicada, Escuela Graduada de Trabajo Social Beatriz Lassalle, Universidad de Puerto Rico, en Río Piedras. 
Garriga Vidal, Lydimar \& Negrón Rodríguez, Damaris. (2008). La formación neoliberal en el Trabajo Social: análisis de la filosofia, misión, metas, objetivos y contenido de los cursos medulares de la Escuela Graduada de Trabajo Social Beatriz Lassalle a la luz de las políticas neoliberales educativas de: la educación como mercancía y fragmentación del conocimiento y opinión de los(as) estudiantes del programa de maestría. Tesis de maestría no publicada, Escuela Graduada de Trabajo Social Beatriz Lassalle, Universidad de Puerto Rico, en Río Piedras.

Guerra, Yolanda. (2003). Instrumentalidad del proceso de trabajo y Servicio Social. En: Elisabete Borgiani, Yolanda E. Guerra \& Carlos Montaño (Coord.), Servicio Social crítico, hacia la construcción del nuevo proyecto ético-político profesional (pp.153-170). Sao Paulo, Brasil:Cortez Editora.

Guardiola Ortiz, Dagmar. (1998). Trabajo Social en Puerto Rico, ¿Asistencia, desarrollo, transformación? Puerto Rico: Editorial Edil.

Guardiola Ortiz, Dagmar. (Coord.). (2006). El Trabajo Social en el caribe hispano antillano Vol. I, el contexto regional: una perspectiva crítica, La Editorial Universidad de Puerto Rico, San Juan, Puerto Rico.

Guardiola Ortiz, Dagmar. (2006). El Trabajo Social en el caribe hispano antillano Vol. II, un análisis crítico de su situación: retos y perspectivas. San Juan: Editorial Universidad de Puerto Rico.

Honda, María Virginia. (1955) Estudio del Colegio de Trabajadores Sociales de Puerto Rico. Tesis de maestría no publicada, Escuela Graduada de Trabajo Social Beatriz Lassalle, Universidad de Puerto Rico, en Río Piedras.

Iamamoto, Marilda. (1997). Servicio Social y división del trabajo. Sao Paulo: Cortez Editora.

Iamamoto, Marilda. (2003). Servicio Social en la contemporaneidad. Sao Paulo: Cortez Editora. 
Iamamoto, Marilda. (2000). La metodología en el Servicio Social: lineamientos para el debate. En Elizabete Borgiani y Carlos Montaño (Coord.), Metodología y Servicio Social hoy (pp. 93104). Sao Paulo, Brasil: Cortez Editora.

Iamamoto, Marilda. (2003) El debate contemporáneo del Servicio Social y la ética profesional. En Elisabete Borgiani, Yolanda Guerra y Carlos Montaño, (Coord.), Servicio Social crítico, hacia la construcción del nuevo proyecto ético-político profesional (pp.249-270). Sao Paulo, Brasil: Cortez Editora.

Lassalle, Beatriz. (1939). El Gobierno de Puerto Rico y el Trabajo Social. Revista de Servicio Social, 1(1).

Lessa, Sergio. (2000). El método y su funcionamiento ontológico. En Elizabete Borgiani y Carlos Montaño (Coord.), Metodología y Servicio Social hoy (pp.199-228). Sao Paulo, Brasil: Cortez Editora.

López, Krimilda \& Lugo de Toro, Doris. (1961). Conocimiento y actitudes de número de médicos sobre las funciones de los trabajadores sociales en agencias gubernamentales de salud en Puerto Rico. Tesis de maestría no publicada, Escuela Graduada de Trabajo Social Beatriz Lassalle, Universidad de Puerto Rico, en Río Piedras.

Lukàcs, György. (2004). Ontología del ser social, el trabajo. Buenos Aires, Argentina: Ediciones Herramienta

Maldonado Díaz, Hilda V.; Muñiz Ảrocho, Bernardo; Ramírez Polo, José R.; Roche Velásquez, Mario; Rodríguez Bernguer, Julia E.; Rosa Pérez, Lydia M.; Rosado Malpica, Néstor; Saliva Guillot, Sara M.; Sierra Martínez, Adminda; Torres Rivera, Francisco; Vélez Velásquez,Eulogio y Vélez González, Zulma. (1969). Servicio Social en la Puerto Rican Cement Company Incorporada. Tesis de maestría no publicada, Escuela Graduada de Trabajo Social Beatriz Lassalle, Universidad de Puerto Rico, en Río Piedras.

Marx, Carlos. (1946). El capital, tomo I. México: Fondo de Cultura Económica. 
Miranda Villamil, Alba; Morena de Rodríguez, Olga; Otero, Gloria Josefina; Tous Gómez, Ana Isabel y Vélez Hernández, Ligia. (1964). El papel del trabajador social en la acción social. Tesis de maestría no publicada, Escuela Graduada de Trabajo Social Beatriz Lassalle, Universidad de Puerto Rico, en Río Piedras.

Mirayes, Sastre, Aida; Pérez Guardiola, Hilda; Robles González, José y Rodríguez Pérez, Antonio S. (1959). Funciones de los trabajadores medico sociales del Distrito Sanitario de Río Piedras, Puerto Rico. Tesis de maestría no publicada, Escuela Graduada de Trabajo Social Beatriz Lassalle, Universidad de Puerto Rico, en Río Piedras.

Montaño, Carlos. (2000). El debate metodológico de los 80\90. El enfoque ontológico versus el abordaje epistemológico. En Elizabete Borgiani y Carlos Montaño (Coord.), Metodología y Servicio Social (pp.9-34). Sao Paulo, Brasil: Cortés Editora.

Montaño, Carlos. (1998). La naturaleza del Servicio Social, un ensayo sobre su génesis su especificidad y su reproducción. Sao Paulo, Brasil: Cortés Editora.

Netto, José. (1997). Capitalismo Monopolista y Servicio Social. Sao Paolo, Brasil: Cortez Editora.

Netto, José. (2000). Método y teoría en las diferentes matrices del Servicio Social. En Elizabete Borgiani y Carlos Montaño (Coord.), Metodología y Servicio Social (pp.51-92). Sao Paulo, Brasil: Cortez Editora.

Netto, José. (2003). El Servicio Social y la tradición marxista. En Elizabete Borgiani, Yolanda Guerra y Carlos Montaño (Coord.), Servicio Social crítico, hacia la construcción del nuevo proyecto ético-político profesional (pp.153-170). Sao Paulo, Brasil: Cortez Editora.

Noriega de Santa, Carmen Lydia y Crespo de Rosa. Candelaria. (1956). El trabajo social escolar en Puerto Rico. Tesis de maestría no publicada, Escuela Graduada de Trabajo Social Beatriz Lassalle, Universidad de Puerto Rico, en Río Piedras. 
Parra, Gustavo. (2001). Antimodernidad y Trabajo Social, Orígenes y expansión del Trabajo Social Argentino. Buenos Aires: Espacio Editorial.

Pratts, Saúl. (1987). La política social de Puerto Rico. Puerto Rico: Ediciones Porta Coeli.

Pratts, Saúl. (1996). La privatización del pacto social en Puerto Rico. Puerto Rico: Ediciones Porta Coeli.

Quiroga, Consuelo. (2000). Invasión positivista en el marxismo: el caso de la enseñanza de la metodología en el Servicio Social. En Elizabete Borgiani y Carlos Montaño (Coord.), Metodología y Servicio Social (pp. 121-170). Sao Paulo, Brasil: Cortez Editora.

Rodríguez, Norma. (2001) El positivismo. En Perspectivas metodológicas en Trabajo Social. Buenos Aires: Espacio Editorial.

Rojas, Rosa. (2001). Métodos para la investigación social unu proposición dialéctica. México: Plaza y Valdés Editores.

Ruiz, Magali. (1997). La práctica del Trabajo Social, de lo específico a lo genérico, Puerto Rico: Editorial Edil. 